\title{
Integrated Assessment of the Air Quality around the Environs of Dr. Abubakar Sola Saraki Memorial Abattoir, Ilorin, Kwara State, Nigeria
}

\author{
Victor SHEGUN, Oluwatuyi ( Corresponding Author) \\ Environmental Health Science Department \\ Kwara State University \\ Malete, Nigeria \\ Co-Authors \\ Adeola Anthony DADA ${ }^{1}$, Saheed Adesola OGUNTADE ${ }^{2}$, John Tolulope SALAMI ${ }^{3}$, \\ Lateefat Olajumake AYINLA ${ }^{4}$,Mojisola BELLO ${ }^{5}$ Oluwakemi Ifedayo Sam-Ijadele ${ }^{6}$
}

\begin{abstract}
Environmental media of Dr. Abubakar Sola Saraki memorial abattoir, Akerebiata, Ilorin.The quality of air around the abattoir was assessed for PM2.5, PM10, HCHO and Volatile Organic Compounds. Result revealed that analyzed data were above WHO standards, the increase in the values could be due to burning of animal and pollution from animal waste which has deleterious effect of living organisms and system. It was revealed some these pollutants has relative high and low readings in the day and at dawn respectively which is also applicable to the temperature readings. High temperature is favorable to thermophiles biological activities eveidently shown by the outpour of gases. There could be consideration towards re-siting of the abattoir to a different location. Recommendations were proffered which includes: Reduction of pollutants emitted from the abattoir through sensitization of butchers and meat sellers in the abattoir, Government agencies should swing into action to regulate the level of pollutants released into the environment on routine basis while also empower the EHOs to step up routine inspection of Abattoirs to eliminate foreseeable threats to public health.
\end{abstract}

\section{INTRODUCTION}

Air pollution is the release of harmful or toxic substance into the ambient environment which has the ability to alter the natural state of the air and renders it dangerous to man, plant and animals. Environmental media of Dr. Abubakar Sola Saraki memorial abattoir, Akerebiata, Ilorin .The quality of air around the abattoir was assessed for PM2.5, PM10, HCHO and Volatile Organic Compounds. Air pollution is the introduction of substances into the atmosphere that harm or cause discomfort to human and other living and non-living things. Ambient air pollution has constituted serious threat to man and his environment during production processes that emits obnoxious gases into the atmosphere. Adelegan, J.A (2002).
Abattoirs plays major role in the emission of toxic gases due to burning activities in an open space, gases such as Carbon monoxide (CO), Carbon dioxide ( $\mathrm{CO} 2)$ and other volatile organic compounds VOCs are released into the environment[3]. Toxic smokes released from local incinerator may lead to increase release of toxic gases. These Compound are dangerous and emit unpleasant odours. Instead, the sitting of abattoirs is on the rise exponentially, research has shown that air pollution problem in Nigeria is attributed to indiscriminate sitting of abattoir. Abattoir emission also contributes to global problem caused by greenhouse gas emission. Emissions are variable in space and time and in how they interact within the various processes and media affected. Abattoir also consumes fuel for energy production thereby emitting $\mathrm{CO} 2$, NOx, SO2 and particulates. Till date, the extent of detrimental impact of abattoir operation on the ambient air quality are yet an unknown issue in Nigeria.

The most alarming case is the high incidence of chronic lung infection among abattoir operators.

Abattoir are one of industries that releases appreciable amount of organic and inorganic air pollutants such as hydrogen sulphide (H2S), Methanethiol, Ammonia, Di methyl sulphide etc. during the heating of animal tissues. These pollutants with high concentration can be objectionable and pose health effect such as headache, nausea, eye irritation paralysis and even death, therefore it must be controlled. Michael Kymisis etal' 2008

VOCs causes damage to organs such as liver, lung etc while also causing irritation of the throat, nausea, loss of coordination, headache, eye irritation and in most severe cases leads to death.

\section{The Aim of this study is:}

To assess the air quality around Dr. Abubakar Sola Saraki memorial abattoir, Akerebiata, Ilorin. 
$>$ Objective of the study

- To identify the ambient air quality around around Dr. Abubakar Sola Saraki memorial abattoir, Akerebiata, Ilorin.

- To correlate the ambient air quality in the abattoir with activities of the abattoir daily.

- To proffer possible solutions based on findings.

$>$ Description of the Study Area

- Abubarkar Saraki Abattoir, Akerebiata, Ilorin, with Coordinate: Latitudes ( $8^{\circ} 30^{\prime} 0^{\prime} \mathrm{N}$ and Longitudes $4^{\circ} 32^{\prime}$ 60’E).

- Air samples were collected randomly in three different locations with three different readings.

- The air quality index of [PM25, PM10, HCHO and VOCs]

\section{METHODOLOGY AND PROCEDURE}

Samples were collected from four sampling points in Dr. Bukola Saraki Memorial Abbatoir located along sobi road, Ilorin, Kwara State . SP1, SP2, SP3, SP4 were used as code for the points were samples were collected. Data were collected on-site taking into consideration the direction of wind and time table for collection of sample every hour. The samples collected were basically on CO, $\mathrm{CO} 2, \mathrm{H} 2 \mathrm{~S}$, PM10, VOCs, PM25 and HCHO due to the fact that they are of greater public health concern in the study area. Multi Gas Analyzer [Electrochemical], GM Model $19773 \mathrm{H}$ for VOCs and H2S and MRP (PCM 50) for CO, $\mathrm{CO} 2$ were used in analysis of sample under a complete conditioning system including the reading of the relative humidity temperature and wind speed. For ten days reading was done around 6:25 am to $6 \mathrm{pm}$ within one hour spacing. While taking the readings, there were days when it rained while there were sunny days which has so much impact on the samples collected. The reading were noted immediately and represented in tables while analysis was done to identify air pollutants using mixed effect and random subject effect models repeatedly with the use of logistic and linear models to correlate association between the two. PSI in accordance with EPA was calculated using statistical calculation and model to analyze PSI of the major pollutants. AQI was used to determine the air quality and it was done in comparison with UNESPA standard. $(0-15)=$ Very Good, $(16-31)$ AQI = Good, $(32-49)=$ Fair, $(50-$ 99) $\mathrm{AQI}=$ Poor $(100+=$ Very Poor.

\section{RESULT AND DISCUSSIONS AIR QUALITY MEASUREMENT OF DR. ABUBAKAR SOLA SARAKI MEMORIAL ABATTOIR, ALONG SOBI ROAD, ILORIN KWARA STATE}

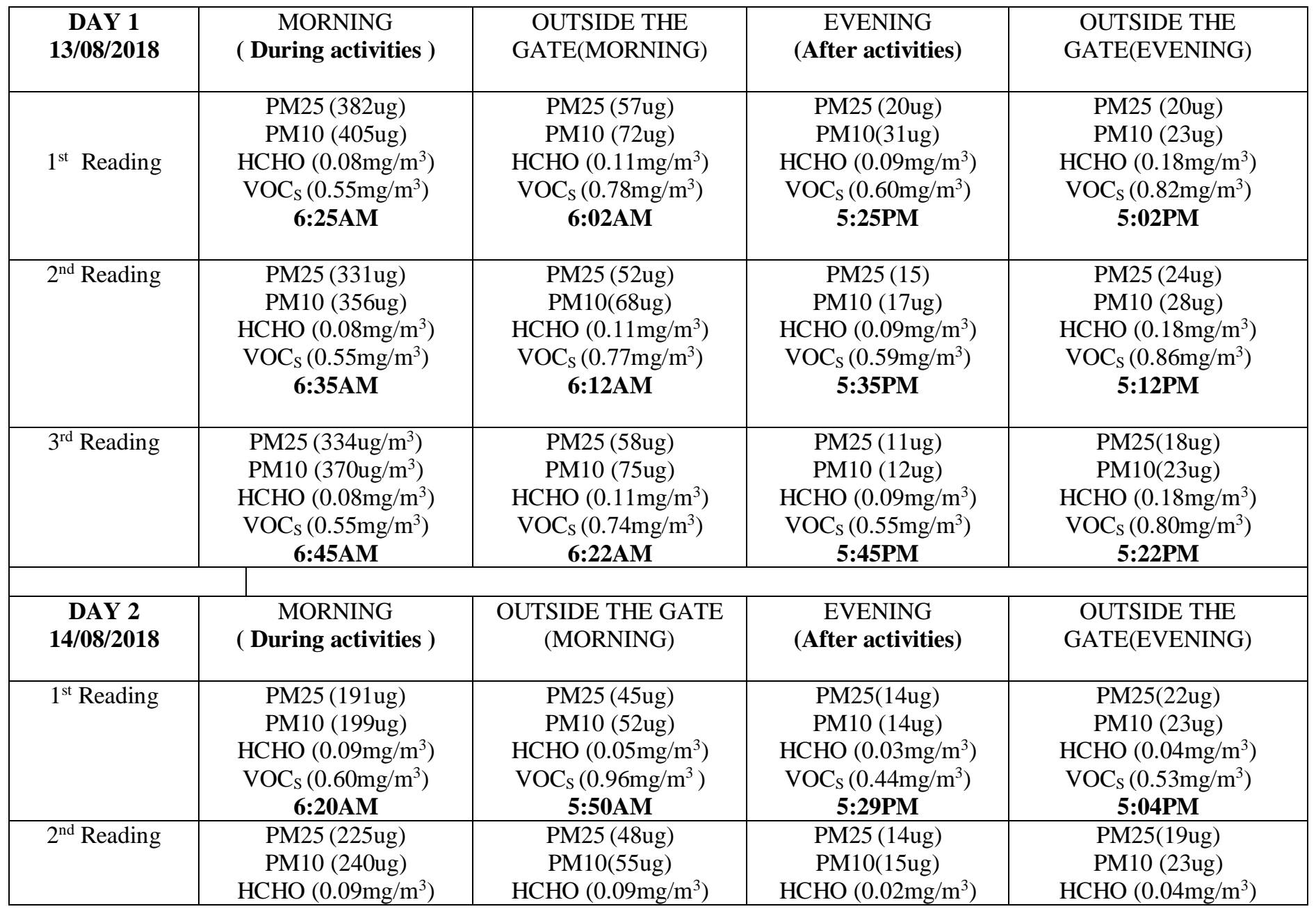


ISSN No:-2456-2165

\begin{tabular}{|c|c|c|c|c|}
\hline & $\begin{array}{c}\mathrm{VOC}_{\mathrm{S}}\left(0.59 \mathrm{mg} / \mathrm{m}^{3}\right) \\
\mathbf{6 : 3 0 A M}\end{array}$ & $\begin{array}{c}\mathrm{VOC}_{\mathrm{S}}\left(0.98 \mathrm{mg} / \mathrm{m}^{3}\right) \\
\text { 6:00AM }\end{array}$ & $\begin{array}{c}\text { VOC }_{\mathrm{S}}\left(0.44 \mathrm{mg} / \mathrm{m}^{3}\right) \\
\mathbf{5 : 3 9 P M}\end{array}$ & $\begin{array}{c}\operatorname{VOC}_{\mathrm{S}}\left(0.53 \mathrm{mg} / \mathrm{m}^{3}\right) \\
\mathbf{5 : 1 4 P M}\end{array}$ \\
\hline $3^{\text {rd }}$ Reading & $\begin{array}{c}\text { PM25 (147ug) } \\
\text { PM10 (154ug) } \\
\text { HCHO }\left(0.09 \mathrm{mg} / \mathrm{m}^{3}\right) \\
\text { VOC }_{S}\left(0.55 \mathrm{mg} / \mathrm{m}^{3}\right) \\
\mathbf{6 : 4 0 A M}\end{array}$ & $\begin{array}{c}\text { PM25(50ug) } \\
\text { PM10 (60ug) } \\
\text { HCHO }\left(0.09 \mathrm{mg} / \mathrm{m}^{3}\right) \\
\text { VOC }_{\mathrm{S}}\left(0.94 \mathrm{mg} / \mathrm{m}^{3}\right) \\
\mathbf{6 : 1 0 A M}\end{array}$ & $\begin{array}{c}\text { PM25 (16ug) } \\
\text { PM10 (20ug) } \\
\text { HCHO }\left(0.03 \mathrm{mg} / \mathrm{m}^{3}\right) \\
\text { VOCS }\left(0.44 \mathrm{mg} / \mathrm{m}^{3}\right) \\
\mathbf{5 : 4 9 P M}\end{array}$ & $\begin{array}{c}\text { PM25(22ug) } \\
\text { PM10 }(25 \mathrm{ug}) \\
\text { HCHO }\left(0.04 \mathrm{mg} / \mathrm{m}^{3}\right) \\
\text { VOC }_{S}\left(0.53 \mathrm{mg} / \mathrm{m}^{3}\right) \\
\mathbf{5 : 2 4 P M}\end{array}$ \\
\hline $\begin{array}{c}\text { DAY 3 } \\
15 / 08 / 2018\end{array}$ & $\begin{array}{c}\text { MORNING } \\
\text { ( During activities ) }\end{array}$ & $\begin{array}{c}\text { OUTSIDE THE } \\
\text { GATE(MORNING) }\end{array}$ & $\begin{array}{c}\text { EVENING } \\
\text { (After activities) }\end{array}$ & $\begin{array}{c}\text { OUTSIDE THE } \\
\text { GATE(EVENING) }\end{array}$ \\
\hline $1^{\text {st }}$ Reading & $\begin{array}{c}\text { PM25 (108ug) } \\
\text { PM10(118ug) } \\
\text { HCHO }\left(0.03 \mathrm{mg} / \mathrm{m}^{3}\right) \\
\text { VOC }_{S}\left(0.48 \mathrm{mg} / \mathrm{m}^{3}\right) \\
\mathbf{6 : 2 5 A M}\end{array}$ & $\begin{array}{c}\text { PM25 (67ug) } \\
\text { PM10 (84ug) } \\
\text { HCHO }\left(0.05 \mathrm{mg} / \mathrm{m}^{3}\right) \\
\text { VOC }_{\mathrm{s}}\left(0.55 \mathrm{mg} / \mathrm{m}^{3}\right) \\
\mathbf{6 : 0 0 A M}\end{array}$ & $\begin{array}{c}\text { PM25 (10ug) } \\
\text { PM10 (12ug) } \\
\text { HCHO }\left(0.07 \mathrm{mg} / \mathrm{m}^{3}\right) \\
\text { VOC }_{\mathrm{S}}\left(0.43 \mathrm{mg} / \mathrm{m}^{3}\right) \\
\mathbf{5 : 2 0 P M}\end{array}$ & $\begin{array}{c}\text { PM25 (15ug) } \\
\text { PM10 (16ug) } \\
\text { HCHO }\left(0.03 \mathrm{mg} / \mathrm{m}^{3}\right) \\
\text { VOC }\left(0.57 \mathrm{mg} / \mathrm{m}^{3}\right) \\
\mathbf{5 : 0 0 P M}\end{array}$ \\
\hline $2^{\text {nd }}$ Reading & $\begin{array}{c}\text { PM25(113ug) } \\
\text { PM10 }(127 \mathrm{ug}) \\
\text { HCHO }\left(0.04 \mathrm{mg} / \mathrm{m}^{3}\right) \\
\text { VOC }_{S}\left(0.58 \mathrm{mg} / \mathrm{m}^{3}\right) \\
\mathbf{6 : 3 5} \mathbf{A M}\end{array}$ & $\begin{array}{c}\text { PM25 (64ug) } \\
\text { PM10(78ug) } \\
\text { HCHO }\left(0.05 \mathrm{mg} / \mathrm{m}^{3}\right) \\
\text { VOC }_{\mathrm{S}}\left(0.53 \mathrm{mg} / \mathrm{m}^{3}\right) \\
\mathbf{6 : 1 0 A M}\end{array}$ & $\begin{array}{c}\text { PM25 (20ug/) } \\
\text { PM10(31ug) } \\
\text { HCHO }\left(0.09 \mathrm{mg} / \mathrm{m}^{3}\right) \\
\text { VOC }_{S}\left(0.43 \mathrm{mg} / \mathrm{m}^{3}\right) \\
\mathbf{5 : 3 0 P M}\end{array}$ & $\begin{array}{c}\text { PM25 (19ug) } \\
\text { PM10 (30ug) } \\
\text { HCHO }\left(0.04 \mathrm{mg} / \mathrm{m}^{3}\right) \\
\text { VOC }_{S}\left(0.61 \mathrm{mg} / \mathrm{m}^{3}\right) \\
\mathbf{5 : 1 0 P M}\end{array}$ \\
\hline $3^{\text {rd }}$ Reading & $\begin{array}{c}\text { PM25 (118ug) } \\
\text { PM10 }(128 \mathrm{ug}) \\
\text { HCHO }\left(0.03 \mathrm{mg} / \mathrm{m}^{3}\right) \\
\text { VOCS }_{(}\left(0.48 \mathrm{mg} / \mathrm{m}^{3}\right) \\
\mathbf{6 : 4 5 A M}\end{array}$ & $\begin{array}{c}\text { PM25 (66ug) } \\
\text { PM10 (88ug) } \\
\text { HCHO }\left(0.04 \mathrm{mg} / \mathrm{m}^{3}\right) \\
\text { VOC }_{S}\left(0.55 \mathrm{mg} / \mathrm{m}^{3}\right) \\
\mathbf{6 : 2 0 A M}\end{array}$ & $\begin{array}{c}\text { PM25 (16ug) } \\
\text { PM10 }(16 \mathrm{ug}) \\
\text { HCHO }\left(0.04 \mathrm{mg} / \mathrm{m}^{3}\right) \\
\text { VOCs }\left(0.40 \mathrm{mg} / \mathrm{m}^{3}\right) \\
\mathbf{5 : 4 0 P M}\end{array}$ & $\begin{array}{c}\text { PM25 (18ug) } \\
\text { PM10 }(28 \mathrm{ug}) \\
\text { HCHO }\left(0.04 \mathrm{mg} / \mathrm{m}^{3}\right) \\
\text { VOCs }\left(0.57 \mathrm{mg} / \mathrm{m}^{3}\right) \\
\mathbf{5 : 1 5 P M}\end{array}$ \\
\hline $\begin{array}{c}\text { DAY } 4 \\
16 / 08 / 2018\end{array}$ & $\begin{array}{c}\text { MORNING } \\
\text { ( During activities ) }\end{array}$ & $\begin{array}{c}\text { OUTSIDE THE } \\
\text { GATE(MORNING) }\end{array}$ & $\begin{array}{c}\text { EVENING } \\
\text { (After activities) }\end{array}$ & $\begin{array}{c}\text { OUTSIDE THE } \\
\text { GATE(EVENING) }\end{array}$ \\
\hline $1^{\text {st }}$ Reading & $\begin{array}{c}\text { PM25 (150ug) } \\
\text { PM10 (179ug) } \\
\text { HCHO }\left(0.07 \mathrm{mg} / \mathrm{m}^{3}\right) \\
\text { VOC }_{\mathrm{S}}\left(0.57 \mathrm{mg} / \mathrm{m}^{3}\right) \\
\mathbf{6 : 2 0 A M}\end{array}$ & $\begin{array}{c}\text { PM25 (57ug) } \\
\text { PM10 (75ug) } \\
\text { HCHO }\left(0.09 \mathrm{mg} / \mathrm{m}^{3}\right) \\
\text { VOC }_{\mathrm{s}}\left(0.65 \mathrm{mg} / \mathrm{m}^{3}\right) \\
\mathbf{5 : 5 5 A M}\end{array}$ & $\begin{array}{c}\text { PM25 (23ug) } \\
\text { PM10 }(25 \mathrm{ug}) \\
\text { HCHO }\left(0.02 \mathrm{mg} / \mathrm{m}^{3}\right) \\
\text { VOC }_{\mathrm{S}}\left(0.49 \mathrm{mg} / \mathrm{m}^{3}\right) \\
\mathbf{5 : 1 5 P M}\end{array}$ & $\begin{array}{c}\text { PM25 (28ug) } \\
\text { PM10 (30ug) } \\
\text { HCHO }\left(0.08 \mathrm{mg} / \mathrm{m}^{3}\right) \\
\text { VOC }_{\mathrm{S}}\left(0.90 \mathrm{mg} / \mathrm{m}^{3}\right) \\
\mathbf{4 : 5 5 P M}\end{array}$ \\
\hline $2^{\text {nd }}$ Reading & $\begin{array}{c}\text { PM25 (159ug) } \\
\text { PM10 (184ug) } \\
\text { HCHO }\left(0.07 \mathrm{mg} / \mathrm{m}^{3}\right) \\
\text { VOC }_{\mathrm{S}}\left(0.60 \mathrm{mg} / \mathrm{m}^{3}\right) \\
\mathbf{6 : 3 0 A M}\end{array}$ & $\begin{array}{c}\text { PM25 (54ug) } \\
\text { PM10 (73ug) } \\
\text { HCHO }\left(0.09 \mathrm{mg} / \mathrm{m}^{3}\right) \\
\text { VOC }_{\mathrm{s}}\left(0.57 \mathrm{mg} / \mathrm{m}^{3}\right) \\
\mathbf{6 : 0 5} \mathrm{AM}\end{array}$ & $\begin{array}{c}\text { PM25 (34ug) } \\
\text { PM10 34ug) } \\
\text { HCHO }\left(0.02 \mathrm{mg} / \mathrm{m}^{3}\right) \\
\text { VOC }_{\mathrm{S}}\left(0.50 \mathrm{mg} / \mathrm{m}^{3}\right) \\
\mathbf{5 : 2 5 P M}\end{array}$ & $\begin{array}{c}\text { PM25 (19ug/) } \\
\text { PM10 }(28 \mathrm{ug}) \\
\text { HCHO }\left(0.08 \mathrm{mg} / \mathrm{m}^{3}\right) \\
\text { VOC }_{\mathrm{S}}\left(0.70 \mathrm{mg} / \mathrm{m}^{3}\right) \\
\mathbf{5 : 0 5 P M}\end{array}$ \\
\hline $3^{\text {rd }}$ Reading & $\begin{array}{c}\text { PM25 (217ug) } \\
\text { PM10 (320ug) } \\
\text { HCHO }\left(0.03 \mathrm{mg} / \mathrm{m}^{3}\right) \\
\text { VOC }_{\mathrm{S}}\left(0.60 \mathrm{mg} / \mathrm{m}^{3}\right) \\
\mathbf{6 : 4 0 A M}\end{array}$ & $\begin{array}{c}\text { PM25(60ug) } \\
\text { PM10 (81ug) } \\
\text { HCHO }\left(0.08 \mathrm{mg} / \mathrm{m}^{3}\right) \\
\text { VOC }_{\mathrm{S}}\left(0.61 \mathrm{mg} / \mathrm{m}^{3}\right) \\
\text { 6:15AM }\end{array}$ & $\begin{array}{c}\text { PM25 (37ug) } \\
\text { PM10 (39ug) } \\
\text { HCHO }\left(0.03 \mathrm{mg} / \mathrm{m}^{3}\right) \\
\text { VOC }_{S}\left(0.42 \mathrm{mg} / \mathrm{m}^{3}\right) \\
\mathbf{5 : 3 5 P M}\end{array}$ & $\begin{array}{c}\text { PM25 (21ug) } \\
\text { PM10 (30ug) } \\
\text { HCHO }\left(0.08 \mathrm{mg} / \mathrm{m}^{3}\right) \\
\text { VOC }_{S}\left(0.78 \mathrm{mg} / \mathrm{m}^{3}\right) \\
\mathbf{5 : 1 0 P M}\end{array}$ \\
\hline $\begin{array}{c}\text { DAY } 5 \\
17 / 08 / 2018\end{array}$ & $\begin{array}{c}\text { MORNING } \\
\text { ( During activities ) }\end{array}$ & $\begin{array}{c}\text { OUTSIDE THE } \\
\text { GATE(MORNING) }\end{array}$ & $\begin{array}{c}\text { EVENING } \\
\text { (After activities) }\end{array}$ & $\begin{array}{c}\text { OUTSIDE THE } \\
\text { GATE(EVENING) }\end{array}$ \\
\hline $1^{\text {st }}$ Reading & $\begin{array}{c}\text { PM25 (219ug) } \\
\text { PM10 (289ug) } \\
\text { HCHO }\left(0.07 \mathrm{mg} / \mathrm{m}^{3}\right) \\
\text { VOC }_{\mathrm{S}}\left(0.58 \mathrm{mg} / \mathrm{m}^{3}\right) \\
\mathbf{6 : 2 0 A M}\end{array}$ & $\begin{array}{c}\text { PM25 (35ug) } \\
\text { PM10 (41ug) } \\
\text { HCHO }\left(0.08 \mathrm{mg} / \mathrm{m}^{3}\right) \\
\text { VOC }_{\mathrm{S}}\left(0.63 \mathrm{mg} / \mathrm{m}^{3}\right) \\
\mathbf{5 : 5 0 A M}\end{array}$ & $\begin{array}{c}\text { PM25 (33ug) } \\
\text { PM10 (49ug) } \\
\text { HCHO }\left(0.03 \mathrm{mg} / \mathrm{m}^{3}\right) \\
\text { VOC }_{S}\left(0.48 \mathrm{mg} / \mathrm{m}^{3}\right) \\
\mathbf{5 : 2 5 P M}\end{array}$ & $\begin{array}{c}\text { PM25 (19ug) } \\
\text { PM10 (23ug) } \\
\text { HCHO }\left(0.13 \mathrm{mg} / \mathrm{m}^{3}\right) \\
\text { VOC }_{S}\left(0.80 \mathrm{mg} / \mathrm{m}^{3}\right) \\
\mathbf{5 : 0 0 P M}\end{array}$ \\
\hline $2^{\text {nd }}$ Reading & $\begin{array}{c}\text { PM25 (220ug) } \\
\text { PM10(310ug) } \\
\text { HCHO }\left(0.07 \mathrm{mg} / \mathrm{m}^{3}\right) \\
\text { VOC }_{\mathrm{S}}\left(0.59 \mathrm{mg} / \mathrm{m}^{3}\right) \\
\mathbf{6 : 3 0 A M}\end{array}$ & $\begin{array}{c}\text { PM25 (37ug) } \\
\text { PM10(48ug) } \\
\text { HCHO }\left(0.08 \mathrm{mg} / \mathrm{m}^{3}\right) \\
\text { VOC }_{\mathrm{S}}\left(0.64 \mathrm{mg} / \mathrm{m}^{3}\right) \\
\mathbf{6 : 0 0 a m}\end{array}$ & $\begin{array}{c}\text { PM25(30ug) } \\
\text { PM10(48ug) } \\
\text { HCHO }\left(0.03 \mathrm{mg} / \mathrm{m}^{3}\right) \\
\text { VOC }_{\mathrm{S}}\left(0.54 \mathrm{mg} / \mathrm{m}^{3}\right) \\
\mathbf{5 : 3 5 P M}\end{array}$ & $\begin{array}{c}\text { PM25 (20ug) } \\
\text { PM10(31ug) } \\
\text { HCHO }\left(0.13 \mathrm{mg} / \mathrm{m}^{3}\right) \\
\text { VOC }_{\mathrm{S}}\left(0.85 \mathrm{mg} / \mathrm{m}^{3}\right) \\
\mathbf{5 : 1 0 P M}\end{array}$ \\
\hline $3^{\text {rd }}$ Reading & PM25 (211ug) & PM25 (28ug) & PM25 (35ug) & PM25 (18ug) \\
\hline
\end{tabular}


ISSN No:-2456-2165

\begin{tabular}{|c|c|c|c|c|}
\hline & $\begin{array}{c}\text { PM10 }(278 \mathrm{ug}) \\
\text { HCHO }\left(0.07 \mathrm{mg} / \mathrm{m}^{3}\right) \\
\operatorname{VOC}_{S}\left(0.56 \mathrm{mg} / \mathrm{m}^{3}\right) \\
\mathbf{6 : 4 0 A M} \\
\end{array}$ & $\begin{array}{c}\text { PM10 }(31 \mathrm{ug}) \\
\text { HCHO }\left(0.08 \mathrm{mg} / \mathrm{m}^{3}\right) \\
\text { VOC }_{S}\left(0.56 \mathrm{mg} / \mathrm{m}^{3}\right) \\
\mathbf{6 : 1 0 A M} \\
\end{array}$ & $\begin{array}{c}\text { PM10(44ug) } \\
\text { HCHO }\left(0.03 \mathrm{mg} / \mathrm{m}^{3}\right) \\
\text { VOC }_{\mathrm{S}}\left(0.50 \mathrm{mg} / \mathrm{m}^{3}\right) \\
\mathbf{5 : 4 5 P M} \\
\end{array}$ & $\begin{array}{c}\text { PM10(23ug) } \\
\text { HCHO }\left(0.13 \mathrm{mg} / \mathrm{m}^{3}\right) \\
\text { VOC }_{\mathrm{S}}\left(0.85 \mathrm{mg} / \mathrm{m}^{3}\right) \\
\mathbf{5 : 2 0 P M} \\
\end{array}$ \\
\hline $\begin{array}{c}\text { DAY } 6 \\
18 / 08 / 2018\end{array}$ & $\begin{array}{c}\text { MORNING } \\
\text { ( During activities ) }\end{array}$ & $\begin{array}{c}\text { OUTSIDE THE } \\
\text { GATE(MORNING) }\end{array}$ & $\begin{array}{c}\text { EVENING } \\
\text { (After activities) }\end{array}$ & $\begin{array}{c}\text { OUTSIDE THE } \\
\text { GATE(EVENING) }\end{array}$ \\
\hline $1^{\text {st }}$ Reading & $\begin{array}{c}\text { PM25 (280ug) } \\
\text { PM10 (304ug) } \\
\text { HCHO }\left(0.20 \mathrm{mg} / \mathrm{m}^{3}\right) \\
\text { VOC }_{S}\left(0.52 \mathrm{mg} / \mathrm{m}^{3}\right) \\
\mathbf{5 : 2 5 A M}\end{array}$ & $\begin{array}{c}\text { PM25(43ug) } \\
\text { PM10(54ug) } \\
\text { HCHO }\left(0.17 \mathrm{mg} / \mathrm{m}^{3}\right) \\
\text { VOC }_{\mathrm{S}}\left(0.47 \mathrm{mg} / \mathrm{m}^{3}\right) \\
\mathbf{5 : 0 2 A M}\end{array}$ & $\begin{array}{c}\text { PM25 (30ug) } \\
\text { PM10(34ug) } \\
\text { HCHO }\left(0.00 \mathrm{mg} / \mathrm{m}^{3}\right) \\
\text { VOC }_{\mathbf{S}}\left(0.15 \mathrm{mg} / \mathrm{m}^{3}\right) \\
\mathbf{6 : 1 5 P M}\end{array}$ & $\begin{array}{c}\text { PM25 }\left(29 \mathrm{ug} / \mathrm{m}^{3}\right) \\
\text { PM10(33ug) } \\
\text { HCHO }\left(0.01 \mathrm{mg} / \mathrm{m}^{3}\right) \\
\text { VOC }_{\mathbf{S}}\left(0.17 \mathrm{mg} / \mathrm{m}^{3}\right) \\
\mathbf{4 : 5 0 P M}\end{array}$ \\
\hline $2^{\text {nd }}$ Reading & $\begin{array}{c}\text { PM 25(250ug) } \\
\text { PM10 }(287 \mathrm{ug}) \\
\text { HCHO }\left(0.00 \mathrm{mg} / \mathrm{m}^{3}\right) \\
\text { VOC }\left(0.51 \mathrm{mg} / \mathrm{m}^{3}\right) \\
\mathbf{5 : 3 5 A M}\end{array}$ & $\begin{array}{c}\text { PM25 (49ug) } \\
\text { PM10 (60ug) } \\
\text { HCHO }\left(0.18 \mathrm{mg} / \mathrm{m}^{3}\right) \\
\text { VOC }_{S}\left(0.54 \mathrm{mg} / \mathrm{m}^{3}\right) \\
\mathbf{5 : 1 2 A M}\end{array}$ & $\begin{array}{c}\text { PM25 (28ug) } \\
\text { PM10 }(28 \mathrm{ug}) \\
\text { HCHO }\left(0.14 \mathrm{mg} / \mathrm{m}^{3}\right) \\
\text { VOCS }_{\left(0.49 \mathrm{mg} / \mathrm{m}^{3}\right)} \\
\mathbf{6 : 2 5 P M}\end{array}$ & $\begin{array}{c}\text { PM25 (30ug) } \\
\text { PM10(32ug) } \\
\text { HCHO }\left(0.01 \mathrm{mg} / \mathrm{m}^{3}\right) \\
\text { VOCs }\left(0.17 \mathrm{mg} / \mathrm{m}^{3}\right) \\
\mathbf{6 : 0 0 P M}\end{array}$ \\
\hline $3^{\text {rd }}$ Reading & $\begin{array}{c}\text { PM25 (300ug) } \\
\text { PM10 (325ug) } \\
\text { HCHO }\left(0.18 \mathrm{mg} / \mathrm{m}^{3}\right) \\
\text { VOC }_{S}\left(0.54 \mathrm{mg} / \mathrm{m}^{3}\right) \\
\mathbf{5 : 4 5 A M}\end{array}$ & $\begin{array}{c}\text { PM25 (48ug) } \\
\text { PM10(59ug) } \\
\text { HCHO }\left(0.18 \mathrm{mg} / \mathrm{m}^{3}\right) \\
\text { VOC }_{\mathrm{S}}\left(0.54 \mathrm{mg} / \mathrm{m}^{3}\right) \\
\mathbf{5 : 2 2 A M}\end{array}$ & $\begin{array}{c}\text { PM25 (32ug) } \\
\text { PM10(35ug) } \\
\text { HCHO }\left(0.00 \mathrm{mg} / \mathrm{m}^{3}\right) \\
\text { VOC }^{3}\left(0.17 \mathrm{mg} / \mathrm{m}^{3}\right) \\
\mathbf{6 : 3 5 P M}\end{array}$ & $\begin{array}{c}\text { PM25 (27ug) } \\
\text { PM10(30ug) } \\
\text { HCHO }\left(0.01 \mathrm{mg} / \mathrm{m}^{3}\right) \\
\text { VOC }_{(}\left(0.17 \mathrm{mg} / \mathrm{m}^{3}\right) \\
\mathbf{6 : 1 0 P M}\end{array}$ \\
\hline $\begin{array}{c}\text { DAY } 7 \\
20 / 08 / 2018\end{array}$ & $\begin{array}{c}\text { MORNING } \\
\text { ( During activities ) }\end{array}$ & $\begin{array}{c}\text { OUTSIDE THE } \\
\text { GATE(MORNING) }\end{array}$ & $\begin{array}{c}\text { EVENING } \\
\text { (After activities) }\end{array}$ & $\begin{array}{c}\text { OUTSIDE THE } \\
\text { GATE(EVENING) }\end{array}$ \\
\hline $1^{\text {st }}$ Reading & $\begin{array}{c}\text { PM25 (172ug) } \\
\text { PM10 (209ug) } \\
\text { HCHO }\left(0.14 \mathrm{mg} / \mathrm{m}^{3}\right) \\
\text { VOC }\left(0.49 \mathrm{mg} / \mathrm{m}^{3}\right) \\
\mathbf{6 : 2 5 A M}\end{array}$ & $\begin{array}{c}\text { PM25 (54ug) } \\
\text { PM10(68ug) } \\
\text { HCHO }\left(0.16 \mathrm{mg} / \mathrm{m}^{3}\right) \\
\text { VOC }_{\text {s }}\left(0.44 \mathrm{mg} / \mathrm{m}^{3}\right) \\
\mathbf{5 : 5 8 A M}\end{array}$ & $\begin{array}{c}\text { PM25 (28ug) } \\
\text { PM10(30ug) } \\
\text { HCHO }\left(0.04 \mathrm{mg} / \mathrm{m}^{3}\right) \\
\text { VOCs }\left(0.49 \mathrm{mg} / \mathrm{m}^{3}\right) \\
\mathbf{5 : 2 4 P M}\end{array}$ & $\begin{array}{c}\text { PM25 (13ug) } \\
\text { PM10(20ug) } \\
\text { HCHO }\left(0.14 \mathrm{mg} / \mathrm{m}^{3}\right) \\
\text { VOC }_{\mathrm{S}}\left(0.90 \mathrm{mg} / \mathrm{m}^{3}\right) \\
\mathbf{5 : 0 0 P M}\end{array}$ \\
\hline $2^{\text {nd }}$ Reading & $\begin{array}{c}\text { PM25 }\left(104 \mathrm{ug} / \mathrm{m}^{3}\right) \\
\text { PM10 }\left(188 \mathrm{ug} / \mathrm{m}^{3}\right) \\
\text { HCHO }\left(0.15 \mathrm{mg} / \mathrm{m}^{3}\right) \\
\text { VOC }\left(0.53 \mathrm{mg} / \mathrm{m}^{3}\right) \\
\mathbf{6 : 3 5 A M}\end{array}$ & $\begin{array}{c}\text { PM25 (52ug) } \\
\text { PM10(64ug) } \\
\text { HCHO }\left(0.16 \mathrm{mg} / \mathrm{m}^{3}\right) \\
\text { VOC }_{\mathrm{s}}\left(0.44 \mathrm{mg} / \mathrm{m}^{3}\right) \\
\mathbf{6 : 1 2 A M}\end{array}$ & $\begin{array}{c}\text { PM25 (20ug) } \\
\text { PM10(29ug) } \\
\text { HCHO }\left(0.04 \mathrm{mg} / \mathrm{m}^{3}\right) \\
\text { VOC }_{\mathbf{S}}\left(0.47 \mathrm{mg} / \mathrm{m}^{3}\right) \\
\mathbf{5 : 3 4 P M}\end{array}$ & $\begin{array}{c}\text { PM25 (19ug) } \\
\text { PM10(25ug) } \\
\text { HCHO }\left(0.14 \mathrm{mg} / \mathrm{m}^{3}\right) \\
\text { VOC }_{S}\left(0.83 \mathrm{mg} / \mathrm{m}^{3}\right) \\
\mathbf{5 : 1 0 P M}\end{array}$ \\
\hline $3^{\text {rd }}$ reading & $\begin{array}{c}\text { PM25 (99ug) } \\
\text { PM10 }\left(145 \mathrm{ug} / \mathrm{m}^{3}\right) \\
\text { HCHO }\left(0.14 \mathrm{mg} / \mathrm{m}^{3}\right) \\
\text { VOCs }\left(0.49 \mathrm{mg} / \mathrm{m}^{3}\right) \\
\text { 6:45AM }\end{array}$ & $\begin{array}{c}\text { PM25 (31ug) } \\
\text { PM10 }\left(37 \mathrm{ug} / \mathrm{m}^{3}\right) \\
\text { HCHO }\left(0.20 \mathrm{mg} / \mathrm{m}^{3}\right) \\
\text { VOC }_{S}\left(0.54 \mathrm{mg} / \mathrm{m}^{3}\right) \\
\mathbf{6 : 2 2 A M}\end{array}$ & $\begin{array}{c}\text { PM25 }(19 \mathrm{ug}) \\
\text { PM10 }\left(30 \mathrm{ug} / \mathrm{m}^{3}\right) \\
\text { HCHO }\left(0.02 \mathrm{mg} / \mathrm{m}^{3}\right) \\
\text { VOCS }\left(0.45 \mathrm{mg} / \mathrm{m}^{3}\right) \\
\mathbf{5 : 4 4 P M}\end{array}$ & $\begin{array}{c}\text { PM25(28ug) } \\
\text { PM10 }\left(32 \mathrm{ug} / \mathrm{m}^{3}\right) \\
\text { HCHO }\left(0.13 \mathrm{mg} / \mathrm{m}^{3}\right) \\
\text { VOCs }\left(0.90 \mathrm{mg} / \mathrm{m}^{3}\right) \\
\mathbf{5 : 2 0 P M}\end{array}$ \\
\hline $\begin{array}{c}\text { DAY 8 } \\
23 / 08 / 2018\end{array}$ & $\begin{array}{c}\text { MORNING } \\
\text { ( During activities ), no } \\
\text { burning at all }\end{array}$ & $\begin{array}{c}\text { OUTSIDE THE } \\
\text { GATE(MORNING) }\end{array}$ & $\begin{array}{c}\text { EVENING } \\
\text { (After activities) }\end{array}$ & $\begin{array}{c}\text { OUTSIDE THE } \\
\text { GATE(EVENING) }\end{array}$ \\
\hline $1^{\text {st }}$ Reading & $\begin{array}{c}\text { PM25 (49ug) } \\
\text { PM10 (60ug) } \\
\text { HCHO }\left(0.06 \mathrm{mg} / \mathrm{m}^{3}\right) \\
\text { VOC }_{\mathrm{S}}\left(0.50 \mathrm{mg} / \mathrm{m}^{3}\right) \\
\mathbf{6 : 1 0 A M}\end{array}$ & $\begin{array}{c}\text { PM25 (39ug) } \\
\text { PM10(45ug) } \\
\text { HCHO }\left(0.08 \mathrm{mg} / \mathrm{m}^{3}\right) \\
\text { VOC }_{\mathrm{s}}\left(0.64 \mathrm{mg} / \mathrm{m}^{3}\right) \\
\mathbf{5 : 4 0 A M}\end{array}$ & $\begin{array}{c}\text { PM25 (21ug) } \\
\text { PM10 }(27 \mathrm{ug}) \\
\text { HCHO }\left(0.01 \mathrm{mg} / \mathrm{m}^{3}\right) \\
\text { VOC }_{\mathrm{S}}\left(0.43 \mathrm{mg} / \mathrm{m}^{3}\right) \\
\mathbf{5 : 3 5 P M}\end{array}$ & $\begin{array}{c}\text { PM25 (29ug) } \\
\text { PM10(40ug) } \\
\text { HCHO }\left(0.05 \mathrm{mg} / \mathrm{m}^{3}\right) \\
\text { VOC }_{\mathrm{S}}\left(0.52 \mathrm{mg} / \mathrm{m}^{3}\right) \\
\mathbf{5 : 0 5 P M}\end{array}$ \\
\hline $2^{\text {nd }}$ Reading & $\begin{array}{c}\text { PM25(46ug) } \\
\text { PM10 (59ug) } \\
\text { HCHO }\left(0.05 \mathrm{mg} / \mathrm{m}^{3}\right) \\
\text { VOC }_{\mathrm{S}}\left(0.48 \mathrm{mg} / \mathrm{m}^{3}\right) \\
\mathbf{6 : 2 0 A M}\end{array}$ & $\begin{array}{c}\text { PM25 (47ug) } \\
\text { PM10(56ug) } \\
\text { HCHO }\left(0.07 \mathrm{mg} / \mathrm{m}^{3}\right) \\
\text { VOC }_{S}\left(0.59 \mathrm{mg} / \mathrm{m}^{3}\right) \\
\mathbf{5 : 5 0 A M}\end{array}$ & $\begin{array}{c}\text { PM25 (32ug) } \\
\text { PM10 (40ug) } \\
\text { HCHO }\left(0.02 \mathrm{mg} / \mathrm{m}^{3}\right) \\
\text { VOC }_{\mathrm{S}}\left(0.49 \mathrm{mg} / \mathrm{m}^{3}\right) \\
\mathbf{5 : 4 5 P M}\end{array}$ & $\begin{array}{c}\text { PM25 (26ug) } \\
\text { PM10(38ug) } \\
\text { HCHO }\left(0.04 \mathrm{mg} / \mathrm{m}^{3}\right) \\
\text { VOC }_{\mathrm{S}}\left(0.49 \mathrm{mg} / \mathrm{m}^{3}\right) \\
\mathbf{5 : 1 5 P M}\end{array}$ \\
\hline $3^{\text {rd }}$ Reading & $\begin{array}{c}\text { PM25 (45ug) } \\
\text { PM10(55ug) } \\
\text { HCHO }\left(0.05 \mathrm{mg} / \mathrm{m}^{3}\right) \\
\text { VOC }_{\mathrm{S}}\left(0.47 \mathrm{mg} / \mathrm{m}^{3}\right)\end{array}$ & $\begin{array}{c}\text { PM25 (44ug) } \\
\text { PM10(55ug) } \\
\text { HCHO }\left(0.07 \mathrm{mg} / \mathrm{m}^{3}\right) \\
\text { VOC }_{\mathrm{S}}\left(0.57 \mathrm{mg} / \mathrm{m}^{3}\right)\end{array}$ & $\begin{array}{c}\text { PM25(38ug) } \\
\text { PM10(44ug) } \\
\mathrm{HCHO}\left(0.02 \mathrm{mg} / \mathrm{m}^{3}\right) \\
\text { VOC }_{\mathrm{S}}\left(0.49 \mathrm{mg} / \mathrm{m}^{3}\right)\end{array}$ & $\begin{array}{c}\text { PM25(14ug) } \\
\text { PM10(19ug) } \\
\text { HCHO }\left(0.04 \mathrm{mg} / \mathrm{m}^{3}\right) \\
\text { VOC }_{\mathrm{S}}\left(0.50 \mathrm{mg} / \mathrm{m}^{3}\right)\end{array}$ \\
\hline
\end{tabular}


ISSN No:-2456-2165

\begin{tabular}{|c|c|c|c|c|}
\hline & 6:30AM & 6:00AM & 5:55PM & 5:25PM \\
\hline $\begin{array}{c}\text { DAY } 9 \\
24 / 08 / 2018\end{array}$ & $\begin{array}{c}\text { MORNING } \\
\text { ( During activities ) no } \\
\text { burning }\end{array}$ & $\begin{array}{l}\text { OUTSIDE THE GATE } \\
\text { (MORNING) }\end{array}$ & $\begin{array}{c}\text { EVENING } \\
\text { (After activities) }\end{array}$ & $\begin{array}{l}\text { OUTSIDE GATE } \\
\text { (EVENING) }\end{array}$ \\
\hline $1^{\text {st }}$ Reading & $\begin{array}{c}\text { PM25 (32ug) } \\
\text { PM10 (33ug) } \\
\text { HCHO }\left(0.04 \mathrm{mg} / \mathrm{m}^{3}\right) \\
\text { VOC }_{\text {S }}\left(0.47 \mathrm{mg} / \mathrm{m}^{3}\right) \\
\mathbf{6 : 3 0 A M}\end{array}$ & $\begin{array}{c}\text { PM25 (24ug) } \\
\text { PM10 }(25 \mathrm{ug}) \\
\text { HCHO }\left(0.08 \mathrm{mg} / \mathrm{m}^{3}\right) \\
\text { VOC }_{S}\left(0.58 \mathrm{mg} / \mathrm{m}^{3}\right) \\
\mathbf{6 : 0 0 A M}\end{array}$ & $\begin{array}{c}\text { PM25 (25ug) } \\
\text { PM10(30ug) } \\
\text { HCHO }\left(0.01 \mathrm{mg} / \mathrm{m}^{3}\right) \\
\text { VOC }_{S}\left(0.43 \mathrm{mg} / \mathrm{m}^{3}\right) \\
\mathbf{5 : 3 7 P M}\end{array}$ & $\begin{array}{c}\text { PM25 (23ug) } \\
\text { PM10 }(28 \mathrm{ug}) \\
\text { HCHO }\left(0.03 \mathrm{mg} / \mathrm{m}^{3}\right) \\
\text { VOC }_{S}\left(0.51 \mathrm{mg} / \mathrm{m}^{3}\right) \\
\mathbf{5 : 0 7 P M}\end{array}$ \\
\hline $2^{\text {nd }}$ Reading & $\begin{array}{c}\text { PM25(44ug) } \\
\text { PM10 (46ug) } \\
\text { HCHO }\left(0.04 \mathrm{mg} / \mathrm{m}^{3}\right) \\
\text { VOC }_{S}\left(0.49 \mathrm{mg} / \mathrm{m}^{3}\right) \\
\mathbf{6 : 4 0 A M}\end{array}$ & $\begin{array}{c}\text { PM25 (20ug) } \\
\text { PM10(21ug) } \\
\text { HCHO }\left(0.07 \mathrm{mg} / \mathrm{m}^{3}\right) \\
\text { VOC }_{S}\left(0.54 \mathrm{mg} / \mathrm{m}^{3}\right) \\
\mathbf{6 : 1 0 A M}\end{array}$ & $\begin{array}{c}\text { PM25 (21ug) } \\
\text { PM10 }(27 \mathrm{ug}) \\
\text { HCHO }\left(0.01 \mathrm{mg} / \mathrm{m}^{3}\right) \\
\text { VOC }_{S}\left(0.43 \mathrm{mg} / \mathrm{m}^{3}\right) \\
\mathbf{5 : 4 7 P M}\end{array}$ & $\begin{array}{c}\text { PM25 }(27 \mathrm{ug}) \\
\text { PM10 }(27 \mathrm{ug}) \\
\text { HCHO }\left(0.03 \mathrm{mg} / \mathrm{m}^{3}\right) \\
\text { VOC }_{S}\left(0.50 \mathrm{mg} / \mathrm{m}^{3}\right) \\
\mathbf{5 : 1 7 P M}\end{array}$ \\
\hline $3^{\text {rd }}$ Reading & $\begin{array}{c}\text { PM25 (34ug) } \\
\text { PM10 }\left(36 \mathrm{ug} / \mathrm{m}^{3}\right) \\
\text { HCHO }\left(0.04 \mathrm{mg} / \mathrm{m}^{3}\right) \\
\text { VOC }_{\mathrm{S}}\left(0.46 \mathrm{mg} / \mathrm{m}^{3}\right) \\
\mathbf{6 : 5 0 A M}\end{array}$ & $\begin{array}{c}\text { PM25 (36ug) } \\
\text { PM10(42ug) } \\
\text { HCHO }\left(0.05 \mathrm{mg} / \mathrm{m}^{3}\right) \\
\text { VOC }_{\mathrm{S}}\left(0.50 \mathrm{mg} / \mathrm{m}^{3}\right) \\
\mathbf{6 : 2 0 A M}\end{array}$ & $\begin{array}{c}\text { PM25 (20ug) } \\
\text { PM10(27ug) } \\
\text { HCHO }\left(0.01 \mathrm{mg} / \mathrm{m}^{3}\right) \\
\text { VOC }_{\mathbf{S}}\left(0.44 \mathrm{mg} / \mathrm{m}^{3}\right) \\
\mathbf{5 : 5 7 P M}\end{array}$ & $\begin{array}{c}\text { PM25 (19ug) } \\
\text { PM10(23ug) } \\
\text { HCHO }\left(0.03 \mathrm{mg} / \mathrm{m}^{3}\right) \\
\text { VOC }_{\mathrm{S}}\left(0.48 \mathrm{mg} / \mathrm{m}^{3}\right) \\
\mathbf{5 : 2 7 P M}\end{array}$ \\
\hline $\begin{array}{c}\text { DAY 10 } \\
25 / 08 / 2018\end{array}$ & $\begin{array}{c}\text { MORNING } \\
\text { ( During activities ) }\end{array}$ & $\begin{array}{c}\text { OUTSIDE THE } \\
\text { GATE(MORNING) }\end{array}$ & $\begin{array}{c}\text { EVENING } \\
\text { (After activities) }\end{array}$ & $\begin{array}{c}\text { OUTSIDE THE } \\
\text { GATE(EVENING) }\end{array}$ \\
\hline $1^{\text {st }}$ Reading & $\begin{array}{c}\text { PM25 (37ug) } \\
\text { PM10 41ug) } \\
\text { HCHO }\left(0.06 \mathrm{mg} / \mathrm{m}^{3}\right) \\
\text { VOC }_{S}\left(0.54 \mathrm{mg} / \mathrm{m}^{3}\right) \\
\mathbf{5 : 5 5 A M}\end{array}$ & $\begin{array}{c}\text { PM25(36ug) } \\
\text { PM10 (39ug) } \\
\text { HCHO }\left(0.07 \mathrm{mg} / \mathrm{m}^{3}\right) \\
\text { VOC }_{\mathrm{S}}\left(0.65 \mathrm{mg} / \mathrm{m}^{3}\right) \\
\mathbf{5 : 3 1 A M}\end{array}$ & $\begin{array}{c}\text { PM25 (18ug) } \\
\text { PM10(18ug) } \\
\text { HCHO }\left(0.01 \mathrm{mg} / \mathrm{m}^{3}\right) \\
\text { VOC }_{\mathrm{S}}\left(0.44 \mathrm{mg} / \mathrm{m}^{3}\right) \\
\mathbf{6 : 1 0 P M}\end{array}$ & $\begin{array}{c}\text { PM25 (28ug) } \\
\text { PM10(33ug) } \\
\text { HCHO }\left(0.06 \mathrm{mg} / \mathrm{m}^{3}\right) \\
\text { VOC }_{\mathrm{S}}\left(0.55 \mathrm{mg} / \mathrm{m}^{3}\right) \\
\mathbf{5 : 4 5 P M}\end{array}$ \\
\hline $2^{\text {nd }}$ Reading & $\begin{array}{c}\text { PM25 (44ug) } \\
\text { PM10 (46ug) } \\
\text { HCHO }\left(0.06 \mathrm{mg} / \mathrm{m}^{3}\right) \\
\text { VOC }_{\mathrm{S}}\left(0.50 \mathrm{mg} / \mathrm{m}^{3}\right) \\
\mathbf{6 : 0 5} \mathbf{A M}\end{array}$ & $\begin{array}{c}\text { PM25 (41ug) } \\
\text { PM10 (50ug) } \\
\text { HCHO }\left(0.07 \mathrm{mg} / \mathrm{m}^{3}\right) \\
\text { VOC }_{\mathrm{S}}\left(0.66 \mathrm{mg} / \mathrm{m}^{3}\right) \\
\mathbf{5 : 4 1 A M}\end{array}$ & $\begin{array}{c}\text { PM25 (19ug) } \\
\text { PM10(24ug) } \\
\text { HCHO }\left(0.01 \mathrm{mg} / \mathrm{m}^{3}\right) \\
\text { VOC }_{\mathrm{S}}\left(0.46 \mathrm{mg} / \mathrm{m}^{3}\right) \\
\mathbf{6 : 2 0 P M}\end{array}$ & $\begin{array}{c}\text { PM25 (27ug) } \\
\text { PM10 }(29 \mathrm{ug}) \\
\mathrm{HCHO}^{2}\left(0.06 \mathrm{mg} / \mathrm{m}^{3}\right) \\
\text { VOC }_{\mathrm{S}}\left(0.54 \mathrm{mg} / \mathrm{m}^{3}\right) \\
\mathbf{5 : 5 5 P M}\end{array}$ \\
\hline $3^{\text {rd }}$ Reading & $\begin{array}{c}\text { PM25(42ug) } \\
\text { PM10(46ug) } \\
\text { HCHO }\left(0.06 \mathrm{mg} / \mathrm{m}^{3}\right) \\
\text { VOC }^{2}\left(0.52 \mathrm{mg} / \mathrm{m}^{3}\right) \\
\mathbf{6 : 1 5 A M}\end{array}$ & $\begin{array}{c}\text { PM25 (27ug) } \\
\text { PM10 (29ug) } \\
\text { HCHO }\left(0.06 \mathrm{mg} / \mathrm{m}^{3}\right) \\
\text { VOC }\left(0.54 \mathrm{mg} / \mathrm{m}^{3}\right) \\
\mathbf{5 : 5 1 A M}\end{array}$ & $\begin{array}{c}\text { PM25 (22ug) } \\
\text { PM10 (31ug) } \\
\text { HCHO }\left(0.01 \mathrm{mg} / \mathrm{m}^{3}\right) \\
\text { VOC }_{\mathrm{s}}\left(0.44 \mathrm{mg} / \mathrm{m}^{3}\right) \\
\mathbf{6 : 3 0 P M}\end{array}$ & $\begin{array}{c}\text { PM25 (38ug) } \\
\text { PM10 (47ug) } \\
\text { HCHO }\left(0.06 \mathrm{mg} / \mathrm{m}^{3}\right) \\
\text { VOC }_{\mathrm{s}}\left(0.57 \mathrm{mg} / \mathrm{m}^{3}\right) \\
\mathbf{6 : 0 5 P M}\end{array}$ \\
\hline
\end{tabular}

Table 1

\section{CONCLUSION AND RECOMMENDATION}

AQI was used to determine the air quality and it was done in comparison with UNESPA standard. $(0-15)=$ Very Good, $(16-31)$ AQI $=$ Good, $(32-49)=$ Fair, $(50-$ 99) $\mathrm{AQI}=$ Poor $(100+=$ Very Poor. Result revealed that analyzed data were above WHO standards, the increase in the values could be due to burning activities and pollution from animal waste which has deleterious effect of living organisms and system. It was revealed some these pollutants has relative high and low readings in the day and at dawn respectively which is also applicable to the temperature readings. High temperature is favorable to thermophiles biological activities evidently shown by the outpour of gases.
Diffusion of gases moves interchangeably to low areas which is traceable to the fact that the wind direction is relatively low which means gases do move very fast but limited when distance is considered. There is decrease in concentration of gases and odour when backing the wind direction leading to continuous emission of pollutants, there is every need to be concerned due to high level of pollutants released into the abattoir environs in which some are above the recommended standards.

\section{Recommendations}

- Reduction of pollutants emitted from the abattoir through sensitization of butchers and meat sellers in the abattoir.

- Government agencies should swing into action to regulate the level of pollutants released into the environment on routine basis. 
- Government should empower the EHOs to step up routine inspection of Abattoirs to eliminate foreseeable threats to public health.

- Further research to assess the water and soil quality around the abattoir and meat quality should be done.

\section{REFERENCES}

[1]. Adelegan, J.A (2002) 'Environmental policy and slaughterhouse waste in Nigeria,

[2]. Proceedings of the $28^{\text {th }}$ WEDC conference Kolkata (Calcutta) India. Pg 3-6

[3]. Adesemoye, A. O., Opere, B. O., \& Makinde, S. C. O. (2006). Microbial Content of abattoir waste water and its contaminated soil in Lagos, Nigeria. African Journal of Biotechnology, 5(20), 1963-1968.

[4]. Air quality and health" www.who.int. Accessed July 2012.

[5]. Air Quality Index (AQI) "A Guide to Air Quality and Your Health". US EPA. December 2011. Accessed August 2012.

[6]. Alo, B (2008) "Contribution of road transportation to environmental degradation in Nigeria's urban cities".

[7]. Paper presented in Lamata Annual National Conference of public transportation in Nigeria.

[8]. David Pennise and Kirk Smith (2010) "Biomass Pollution Basics". WHO, 2010.

[9]. Dung-Gwom J.Y. and Magaji, J.Y (2007) 'The Environmental Health Problems Associated with Solid waste

[10]. Management in Gwagwalada, Abuja. The Abuja journal of Geography and Development. 1(1), pg 110126.

[11]. Katarzyna, R. A., Monkiewicz, J., \& Andrzej, G. (2009). Lead, cadmium, arsenic, copper and zinc contents in hair of cattle living in the area contaminated by a copper smelter in 2006-2008. Bull Vet Inst Pulawy, 53, 703-706.

[12]. Michael Kymisis, and Konstantinos Hadjistavrou (2008). "Short-Term Effects of Air Pollution Levels on Pulmonary Function of Young Adults". The Internet Journal of Pulmonary Medicine $\mathbf{9}$

[13]. Miller K. A., Siscovick D. S., Sheppard L., Shepherd K., Sullivan J. H., Anderson G. L., Kaufman J. D. (2007).

[14]. "Long-term exposure to air pollution and incidence of cardiovascular events in women. [Research Support, N.I.H., ExtramuralResearch Support, U.S. Gov't, NonP.H.S.]". The New England journal of medicine 356.

[15]. Magaj, J.Y and Chup, C.D (2012) 'The Effects of Abattoir Waste on Water Quality in Gwagwalada, Abuja

[16]. Nigeria. Ethiopian journal of Environmental Management (EJEM) Vol 5 no 4.

[17]. Odoemelan, S. A., \& Ajunwa, O. (2008). Heavy Metal Status and Physicochemical Properties of Agricultural Soil amended by short term application of animal manure. Journal of Chemical Society of Nigeria, 30,60-63.
[18]. Olanike, K. A. (2002). Unhygienic operation of a city abattoir in south western Nigeria: Environmental implication. AJEAM/RAGEE, 4(1), 23-28.

[19]. Osibanjo, O. and Adie G.U.(2007) 'Impact of effluent from Bodija abattoir on the physio-chemical parameters of

[20]. Oshunkaye stream in Ibadan city, Nigeria. Retrieved 21 November, 2012. Available on line at http://www.academicjournal.org/AJB

[21]. Patra, R. C., Swarup, D., Naresh, R., Kumar, P., Nandi, D., Shekhar, P., Ali, S. L. (2007). Tail hair as an indicator of environmental exposure of cows to lead and cadmium in different industrial areas.

[22]. Ecotoxicol Environ.,Saf.,66, 127-131.

[23]. World Health Organization (WHO) report "Public Health and Air pollution" 2005“" http://www.cleanairforce.com/docs/GCAFHealthBene fitsPoster.pdf 